\title{
Strategies and Tools for Detection of Protein S-Nitrosylation and S-Sulfhydration
}

\author{
YoungJun Ju1, Ming Fu' ${ }^{1,2}$, Lingyun $\mathrm{Wu}^{1,2}$ and Guangdong Yang ${ }^{1,3 *}$
}

${ }^{1}$ Cardiovascular and Metabolic Research Unit, Lakehead University, ON, Canada

${ }^{2}$ Department of Health Science, Lakehead University, ON, Canada

${ }^{3}$ Department of Chemistry and Biochemistry, Laurentian University, ON, Canada

\begin{abstract}
$\mathrm{H}_{2} \mathrm{~S}$ interacts with the free thiol group in the active cysteine residues from target proteins and forms a hydropersulfide moiety (-SSH), termed as S-sulfhydration, which is similar to nitric oxide (NO) regulation of protein by S-nitrosylation. Protein S-sulfhydration now is proposed to mediate most of $\mathrm{H}_{2} \mathrm{~S}$ bioactivities in various cellular functions. A number of methods have been developed for detection of $S$-nitrosylation and $S$-sulfhydration. Selective recognition of hydropersulfide (SSH) is a main target for the detection of S-sulfhydration. Protein Ssulfhydration can be detected by biotin switch assay, which is developed and further modified based on the detection approaches for protein S-nitrosylation. Here we highlight the different tools for detection of protein S-sulfhydration and S-nitrosylation, and provide strategies for developing new methods to locate the modified cysteine residues.
\end{abstract}

Keywords: S-sulfhydration; S-nitrosylation; $\mathrm{H}_{2} \mathrm{~S}$; NO; Biotin switch assay; Hydropersulfide

Abbreviations: biotin-HPDP: N-[6-(biotinamido) hexyl]-3'-(2'pyridyldithio) propionamide; BSA: Biotin Switch Assay; GAPDH: Glyceraldehydes-3-Phosphate Dehydrogenase; DFO: Deferoxamine; DTT: Dithiothreitol; eNOS: endothelial Nitric Oxide Synthase; $\mathrm{H}_{2} \mathrm{~S}$ : Hydrogen Sulfide; MMTS: Methyl Methanethiosulfonate; MS: Mass Spectrometry; MS/MS: tendem Mass Spectrometry; NO: Nitric Oxide; PLN: Phospholamban; PTEN: Phosphatase and Tensin homolog; SNO: S-Nitrosothiol; SOH: Sulfenic acid; SSH: Persulfide..

\section{Introduction}

$\mathrm{H}_{2} \mathrm{~S}$, as a novel gasotransmitter, is proposed to post-translationally modify proteins by yielding a hydropersulfide moiety (-SSH) in specific cysteine residue(s), termed as $S$-sulfhydration [1]. Since the first study on protein $S$-sulfhydration was reported in 2009 , many proteins have been demonstrated to be the targets for $\mathrm{H}_{2} \mathrm{~S} S$-sulfhydration. $\mathrm{H}_{2} \mathrm{~S}$ improves cellular senescence via $S$-sulfhydration of Keap1 [2], relaxes blood vessels and acts as an endothelium-derived hyperpolarizing factor through $S$-sulfhydration of potassium channels [3], helps DNA damage repair via MEK1 $S$-sulfhydration and induction of poly ADP ribose polymerase activity [4], and stimulates nitric oxide (NO) generation via $S$-sulfhydration of endothelial nitric oxide synthase (eNOS) [5]. Furthermore, many other proteins have also reported to be the targets of Ssulfhydration, including phospholamban, NF- $\mathrm{BB}$, parkin, p66Shc, and transient receptor potential $\mathrm{Ca}_{2}{ }^{+}$channels, etc. [6-10]. NO reacts with the specific proteins leading to the covalent incorporation of a NO moiety into the cysteine residues, a process known as $S$-nitrosylation. Protein $S$-nitrosylation is an important post-translational modification that affects a wide variety of proteins involved in multiple cellular processes, and is emerging as one of the most important mechanisms for NO activities [5]. Protein S-nitrosylation shares many similar properties with protein $S$-sulfhydration, and both of them occur in cysteine residues in the targeted proteins. Diverse detection methods have been developed for detection of protein $S$-nitrosylation. Biotin switch assay (BSA) developed by Jeffrey and Snyder is the first method for detection of protein $S$-nitrosylation [11], which can detect $S$-nitrosylation of cysteine residues by replacing the NO moiety with a detectable biotin derivative, N-[6-(biotinamido) hexyl]-3'(2'-pyridyldithio) propionamide (biotin-HPDP). Replacing the NO moiety with biotin derivative can be detected by immunoblotting with biotin antibody or purified with avidin conjugated beads for further analysis, such as tandem mass spectrometry (MS/MS) and two dimensional (2D) analysis. Based on the BSA method of detection of $S$-nitrosylation, a modified BSA was developed to detect protein using different tag molecules or resin, such as thiol-reactive resin, His-tag resin, gold nanoparticle and fluorescent molecule, etc. These modified BSA provide the advanced analysis of protein $S$-nitrosylation through improvement of probe sensitivity. This review compares the different tools for detection of protein $S$-nitrosylation and $S$-sulfhydration, and describes new strategies of further study on protein $S$-sulfhydration. The strategies and tools for detection of protein $S$-nitrosylation and $S$-sulfhydration would provide strong power and solid platform to identify novel diagnostic markers for human diseases and develop new drug for further treatments.

\section{Current detectIon Methods for S-Nitrosylation}

For the detection of protein $S$-nitrosylation, NO-based strategies are mainly considered for either the direct NO detection or NO switched manner. The sulfur-nitrogen bond in S-nitrosothiol (SNO) is particularly labile, and the lability of NO in SNO is an important factor for the detection of $S$-nitrosylation. Based on this concept, many methods were developed for detection of $S$-nitrosylation.

\section{BSA}

BSA is often used for the detection of protein $S$-nitrosylation. The overview of BSA procedure is shown in Figure 1. First, free thiol groups in proteins are blocked with thiol specific methylthiolating agent,

*Corresponding author: G Yang, Department of Chemistry and Biochemistry, Laurentian University, 935 Ramsey Lake Road, Canada, Tel: (705) 675-1151; Fax: (705) 675-4844; E-mail: gyang2@laurentian.ca

Received: October 01, 2015; Accepted: November 09, 2015; Published November 12 2015

Citation: Ju Y, Fu M, Wu L, Yang G (2015) Strategies and Tools for Detection of Protein S-Nitrosylation and S-Sulfhydration. Biochem Anal Biochem 4: 224. doi:10.4172/21611009.1000224

Copyright: $\odot 2015 \mathrm{Ju}$ Y, et al. This is an open-access article distributed under the terms of the Creative Commons Attribution License, which permits unrestricted use, distribution, and reproduction in any medium, provided the original author and source are credited. 
methyl methanethiosulfonate (MMTS). NO in SNO is decomposed and reduced to free thiol with ascorbate, and then labeled with a thiol-specific biotinylating reagent. Total $S$-nitrosylated proteins can be detected with anti-biotin antibody by immunoblotting. To detect specific protein which is $S$-nitrosylated, biotinylated proteins were purified with avidin conjugated beads and then immunoblotted with specific antibody to visualize specific Snitrosylated protein for analysis. In BSA, biotinylated proteins are considered as the $S$-nitrosylated proteins. By using this method, many proteins have been demonstrated to be Snitrosylated, including c-Src, CLIC4, cyclooxygenase-1, capase-3, etc. [12-15]. There are many advanced methods derived from BSA using with the thiol-specific binding reagents containing different tag molecules or resin, such as thiol-reactive resin, His-tag resin, gold nanoparticle and fluorescent molecule, etc $[16,17]$. Qu et al. developed a novel BSA method by using thiol-reactive iodoTMTsixplex reagents to specifically detect and quantify protein $S$-nitrosylation. With this method, a set of proteins involved in antioxidative signaling and mitochondrial metabolic pathways were indentified as $S$-nitrosylated proteins in responding to lipopolysaccharide stimulation in murine BV-2 microglial cells [18]. With another modified method by which gold nanoparticles are used to simultaneously isolate and enrich for free or modified thiol-containing peptides, protein disulfide isomerase and dual specificity phosphatase 12 were $S$-nitrosylated or $S$-glutathionylated [19]. However, those share similar limitation with original BSA for the detection of $S$-nitrosylation, because all these detection methods rely on the visualizing techniques such as immunoblotting and immnofluorescence. To improve the detection limits from visualizing techniques for BSA, BSA samples can be further applied for analysis by MS/MS. For analysis of BSA sample by MS/MS, protein digestion with enzymes, such as trypsin, is necessary since proper samples for MS/ MS range in mass from 50 to 300,000 daltons. Digested fragments can be analyzed with molecular mass by MS/MS. Therefore, biotinylated cysteine and normal cysteine in fragments can be compared with MS/

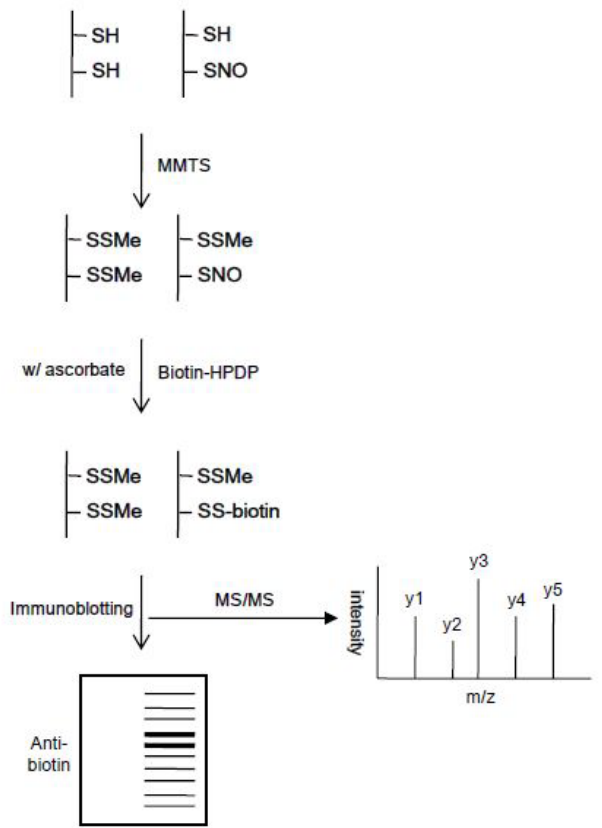

Figure 1: The overview of biotin switch assay for protein S-nitrosylation. The free thiol groups in proteins are firstly blocked with MMTS. SNO group is then decomposed and reduced to free thiol with ascorbate following label with a thiolspecific biotinylating reagent. Total S-nitrosylated proteins can be detected with anti-biotin antibody by immunoblotting or MS/MS analysis.
A

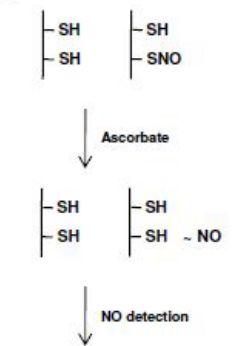

Saville assay

Chemiluminescence

Colorimetry

Fluorescence
B
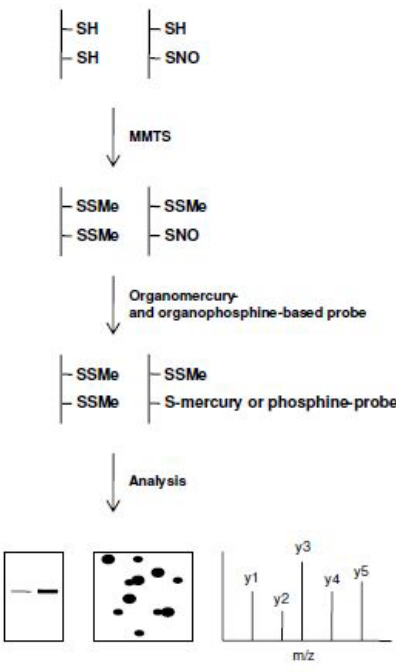

$2 \mathrm{D}$

MSiMS
Figure 2: NO-based strategies for the detection of S-nitrosylation. (A) The Snitrosylated proteins can be incubated with ascorbate to reduce SNO group to free NO, and then other methods, such as saville assay, chemiluminescence, colorimetry, and/or fluorescence, can be used to quantify the generation of NO. (B) The specific reactive probe for detection of S-nitrosylation. Besides with thiol-specific biotinylating reagent, many other probes, including organomercuryand organophosphine-based probe, can be used to detect the SNO group in S-nitrosylated proteins.

MS based on the difference of molecular mass in fragments. The MS/MS analysis also can identify the specific Snitrosylation site on the targeted proteins. With MS/MS analysis, the site of cysteine 139 was clearly identified for $S$-nitrosylation in GAPDH in estrogen-incubated uterine artery endothelial cells [20]. One limitation for MS/MS analysis is hard to quantitatively analyze $S$-nitrosylation. In combination with protein microarray and 2D analysis, MS/MS analysis can readily distinguish the intensity of protein $S$-nitrosylation.

\section{NO based methods for $S$-nitrosylation}

Protein $S$-nitrosylation is a reversible process and can release $\mathrm{NO}$ when the protein is $S$-denitrosylated. This principal is applied to directly detect $\mathrm{NO}$ on $\mathrm{SNO}$-proteins or NO released from SNOproteins $[17,21,22]$. Liberated NO species from SNO can be detected with the common NO detection methods, such as Saville assay, chemiluminescence, colorimetry, and fluorescence, etc. To do so, SNO is firstly reduced by the reagents, such as ascorbate, and then $\mathrm{NO}$ is detected with above mentioned NO measurement assays (Figure 2A). These methods allow the quantitative analysis of protein $S$-nitrosylation; however, these methods are hard to directly identify the specific $S$-nitrosylated site on proteins. In addition, many researchers developed new methods to directly detect NO from Snitrosylated proteins. Organomercury- and organophosphine-based compounds are used for the direct detection of SNO in proteins. After blocking the free thiols on proteins, NO in SNO proteins is replaced with the mercury- and phosphine-based detecting reagents. Detection or capture of $S$-nitrosylation on proteins is based on the fluorescent or resin contained in these reagents. Further analysis can also be performed with immnofluorescent, 2D and MS/MS (Figure 2B). Organomercury- and organophosphine-based compounds are highly selective to SNO, but do not react with sulfur based derivates such as disulfide and oxidized sulfur [23,24]. Furthermore, organophosphine-based compounds possess highly selective reactivity with SNO, not with SSH. 


\section{S-nitrosylated cysteine antibody}

Anti-S-nitrosocyteine antibody is commercially available for detection of protein Snitrosylation. Anti-S-nitrosocysteine antibody directly detects the $S$-nitrosocysteine in $S$-nitrosylated proteins. Anti-Snitrosocyteine antibody can be used for the mapping of $S$-nitrosylated proteosome in the cells, the change of global $S$-nitrosylated proteins in different pathophysiological conditions, and the detection of $S$-nitrosylated proteins from the immunoprecipitated samples with the identified proteins [25-27]. With the application of immunostaining with an anti-S-nitrosocysteine antibody, it was found that C-reactive protein significantly decreases the content of S-nitrosylated proteins as compared with untreated endothelial cells. These data were further confirmed by BSA [28]. $S$-nitrosylated cysteine antibody can be used to detect the global change of protein $S$-nitrosylation, but hardly tell the individual protein.

\section{MS/MS analysis}

MS/MS is an accurate method to detect $S$-nitrosylation and identify the $S$-nitrosylated site on targeted proteins (Figure 3). The lability of the SNO on proteins is the main challenge for MS/MS anaylsis. This challenge can be easily solved by preserving the labile SNO groups with the specific molecules (Figure 3 ), such as thiolating and alkylating reagents $[16,29]$. The labeled SNO can be processed for analysis of protein $S$-nitrosylation by MS/MS. MS is an analytic technique of ionized molecules to determine molecular structure, weight and abundance in biological field. The brief procedure of MS is followed with ionization of a sample, separation of the ionized molecules based on their mass-to-charge ratio, detection of the ionized molecules, and generation of a mass spectrum. Modified protein samples, such as

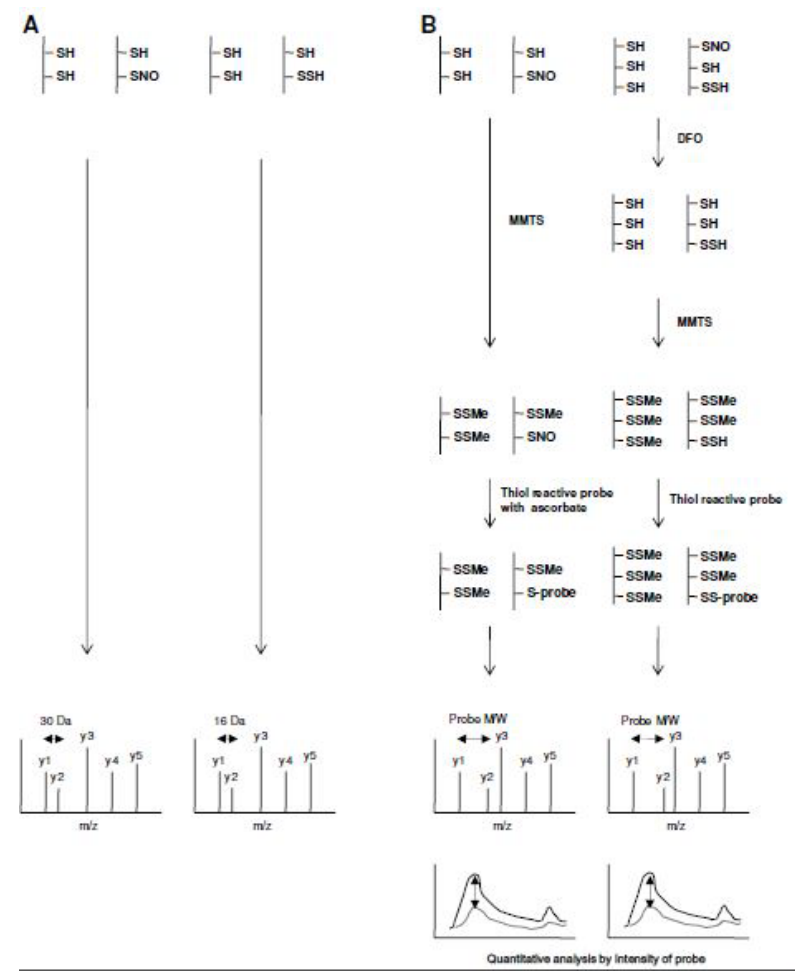

Figure 3: MS/MS analysis of both S-nitrosylation and S-sulfhydration. (A) Direct detection of S-nitrosylation and S-sulfhydration by MS/MS. (B) MS/MS detection of S-nitrosylation and S21 sulfhydration after the modified cysteine residue are tagged or switched with thiol reactive probes.

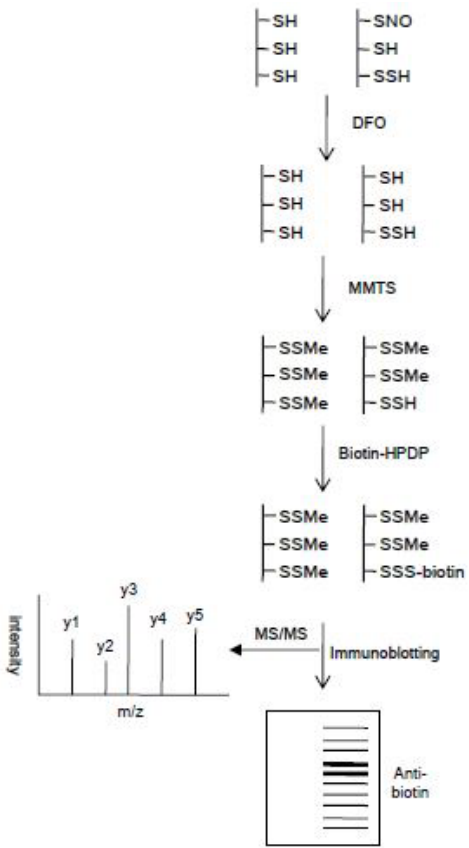

Figure 4: The overview of biotin switch assay for protein S-sulfhydration. The SNO group in proteins are firstly reduced to free $\mathrm{SH}$ group by DFO, and then all free $\mathrm{SH}$ groups are blocked with MMTS following label the $\mathrm{SSH}$ group with a thiol-specific biotinylating reagent. Total S-sulfhydrated proteins can be detected with anti-biotin antibody by immunoblotting or MS/MS analysis.

protein $S$-nitrosylation and $S$-sulfhydration, can be analyzed by MS to detect and identify the modification of proteins relying on the massto-charge shift in protein modification [30]. In protein Snitrosylation, the modification of cysteine residue by $\mathrm{NO}$ increases its mass to $29 \mathrm{Da}$. This mass shift can be detected with optimized instrument and sample preparation by MS even though its difficulty was found with decomposition of mass addition in ionization step [29,31]. In order to avoid this problem, mass addition, such as NO in protein $S$-nitrosylation, is switched to stable molecules by labeling. MS is not only used for the identification of protein modification, but also can be used to quantitativly analyze protein modification with the addition of fluorescent probe to the labeled samples (Figure 3).

\section{Other methods}

Although a number of methods have been developed for detection of $S$-nitrosylation as described above, the quantitative proteomic analysis of $S$-nitrosylation is still a major issue. To determine the change of the $S$-nitrosylated proteins by NO donor, a quantitative proteomic analysis was recently developed in mesangial cells through $2 \mathrm{D}$ analysis [32]. NO-induced or non-induced cell extract was prepared and purified with BSA. Purified $S$-nitrosylated proteins were then separated and visualized by 2D PAGE based on their differential $\mathrm{pH}$ value for the isoelectric point and molecular masses. Quantitative proteomic analysis of proteins $S$-nitrosylation was performed with comparison of spot densitometry between NO-induced and non-induced samples. Identification of $S$-nitrosylated proteins was determined by MALDITOF MS with peptide mass fingerprinting. With BSA in combination of 2D gel electrophoresis and MALDI-TOF MS, 31 novel protein targets of $S$-nitrosylation were identified in NO-treated and cytokine-activated murine mesangial cells [32]. 


\section{Current Detection Methods of Protein S-Sulfhydration}

\section{BSA}

At the present, BSA is employed as a main technique to determine $S$-sulfhydrated proteins (Figure 4). The difference between BSA detection of $S$-sulfhydration and $S$-nitrosylation is in the first step, where deferoxamine (DFO) is used to reduce SNO to free thiol and then all free thiols are blocked by MMTS (Figure 4). All the rest of procedures are same as BSA detection of $S$-nitrosyaltion. The SSH bond in $S$-sulhydrated proteins is more sensitive to oxygen and light, the BSA detection is better to use fresh samples and avoid light. So far many proteins have been proved to be the target of $S$-sulfhydration by $\mathrm{H}_{2} \mathrm{~S}$, including GAPDH, actin, and tubulin, etc [33]. In BSA for $S$-sulfhydration detection, the selectivity of MMTS to free thiol and persulfide in proteins is questioned. Reactivity of MMTS to persulfide was determined for the detection of protein $S$-sulfhydration [34]. The chemical compounds containing free thiol, such as GSH, and proteins, papain and glutathione peroxidase $3(\mathrm{Gpx} 3)$, were tested in this study. The selectivity of MMTS was not detected between free thiol and persulfide in chemical reaction. However, the detection mechanism of $S$-sulfhydration by BSA still remains unclear because it is not well determined in biological complex samples even though the selectivity of MMTS is questionable in chemical reaction. In order to avoid this issue, many studies used another method, such as MS/MS, to confirm protein $S$-sulfhydration along with BSA or have been trying to develop new detection methods for $S$-sulfhydration $[1,8]$. Even though, there is no doubt that BSA is still a powerful tool for the detection of protein $S$-sulfhydration now. When the mechanism of selectivity of MMTS is clearly determined in biological complex samples in the near future, it will provide the fundamental knowledge of regulatory mechanism of protein $S$-sulfhydration in biological system since it may result from the specific motif or regulatory formation of protein Ssulfhydration.

A

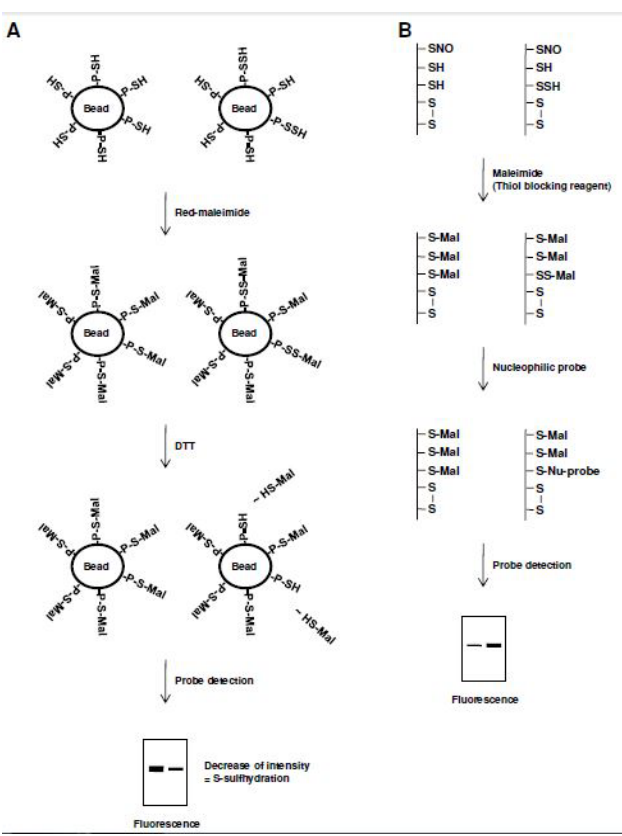

Figure 5: Tag switch assay for S-sulfhydration. (A) Simple tag switch method with red fluorescent maleimide. Maleimide can bind with $\mathrm{SSH}$ and $\mathrm{SH}$ to form SS-maleimide and Smaleimide, and then only SS-maleimide can react with DTT to become free $\mathrm{SH}$ group following quantitative analysis of the change of fluoresce intensity. (B) Advanced tag switch technique to detect S-sulfhydration with the combination of maleimide and nucleophilic probe.

\section{Tag switch assay}

Besides with BSA, simple tag switch assay is recently developed for the detection of protein $S$-sulfhydration [8]. Maleimide can bind with SSH and $\mathrm{SH}$ to form SS-maleimide and Smaleimide, respectively. Immuno-captured proteins with beads are first incubated with fluorescent tagged maleimide (red-maleimide), and then redmaleimide linked to persulfide is removed by dithiothreitol (DTT) incubation. Quantitative analysis of $S$-sulfhydrated protein can be determined with the decrease amount of fluorescent intensity because only SS-maleimide but not S-maleimide can be removed by DTT (Figure 5A). Pyruvate carboxylase and NFkb were indentified as $S$-sulfhydrated proteins by this newly developed tag switch assay $[8,35]$. Thiol-blocking reagent and nucleophilic probe can also be used for the detection of Ssulfhydration. First, free thiols and persulfides are blocked with thiol-blocking reagents, such as maleimide. Nucleophilic probe attacks persufide blocked by thiol-blocking reagents and then replaces thiol blocking reagents linked to persulfide. $S$-sulfhydrated proteins are then detected based on the intensity of probe which is connected to nucleophile. The selectivity of this technique has been proved in purified protein as well as cell lysates [36] (Figure 5B).

\section{MS/MS}

Similar to the detection method of S-nitrosylation, MS/MS is a powerful tool for the identification of particular cysteine residues in $S$-sulfhydrated protein. MS/MS has been used to identify the cysteine residue in S-sulfhydrated GAPDH [1]. Compared with SNO, SSH is more stable, so MS/MS is more appropriate for detection of protein $S$-sulfhydration [5,37]. To improve the sensitivity of MS/MS, the switching and tagging techniques are also employed in MS/MS analysis of protein $S$-sulfhydration [36]. Quantitative proteomic analysis of MS/ MS for Ssulfhydration has not been tested yet.

\section{New Strategies for Detection of Protein S-Sulfhydration}

For the detection of the modified hydropersulfide group in $S$-sulfhydrated cysteine, the main challenge is to selectively recognize the SSH from other sulfur derivates, such as disulfide, SNO, thiol, and oxidized sulfur $[38,39]$.

\section{Advanced tag switch and captured assay}

Development of selective binding reagent for SSH, such as organomercury- and organophosphine-based reagents, will be a great breakthrough for the detection of $S$-sulfhydration. However, there are huge challenges for the development of SSH-selective detection reagent since SSH shares similar properties with disulfide and thiol. To avoid these challenges, two strategies for the detection of $S$-sulfhydration can be employed. First, released $\mathrm{H}_{2} \mathrm{~S}$ by disulfide reducing reagents containing no sulfur, such as tris ( 2 carboxyethyl)phosphine (TCEP), can be detected from $S$-sulfhydration with common $\mathrm{H}_{2} \mathrm{~S}$ detection methods (Figure 6A). Second, the disulfide bond in $S$-sulfhydration is used for the release of thiol group (Figure 6B). Briefly, free thiols are blocked with thiol blocking reagents which sulfur is not contained, such as maleimide. The maleimide bound to persulfide is broken by TCEP in order to release thiol-maleimide from S-sulfhydration. Released thiolmaleimide is then detected by the use of thiol reactive probe which can be activated by reaction with thiols. Third, thiol reactive beads can be used to establish the detection methods for $S$-sulfhydration (Figure 6C). $S$-nitrosylation is reduced by DFO and free thiols are then blocked by MMTS in proteins. Persulfide in proteins is pull-downed with thiol reactive beads. Ssulfhydrated proteins are eluted from beads with TCEP treatment. We tested this method with GAPDH and visualized with 
immunoblotting, and the data clearly showed NaHS treatment induces GAPDH S-sulfhydration, which confirms the results with BSA method.

\section{$S$-sulfhydrated cysteine antibody}

Anti-SSH cysteine antibody can also be designed and developed

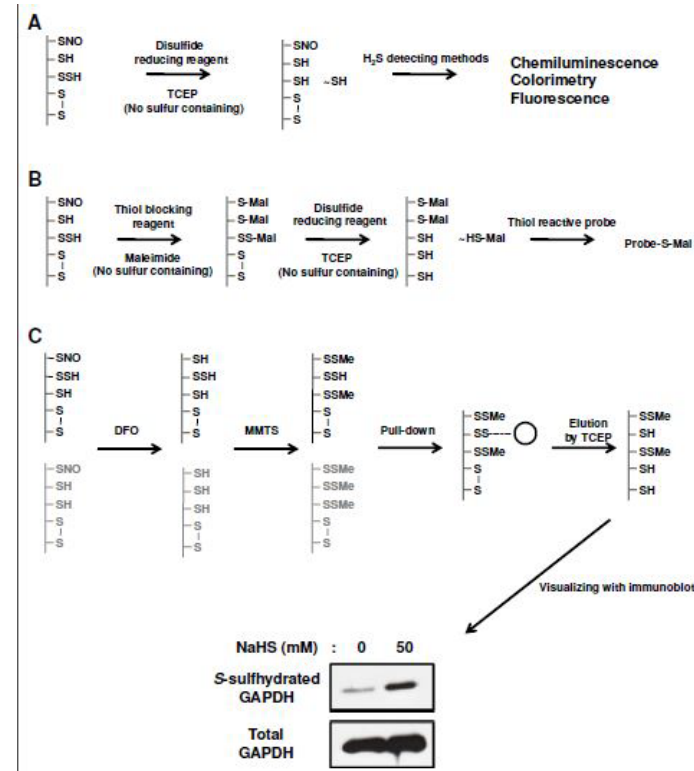

Figure 6: Proposed detection methods for S-sulfhydration. (A) The detection method based on released $\mathrm{H} 2 \mathrm{~S}$ from S-sulfhydrated protein. (B) The detection method of S-sulfhydration after switched with thiol reactive probe (C) The detection method of S-sulfhydration with thiol reactive probe. HepG2 cells were harvested after incubation with NaHS $(50 \mu \mathrm{M})$ for 2 hours, and then homogenized in HEN buffer (250 mM HEPES (pH 7.7), $1 \mathrm{mM}$ EDTA, and $0.1 \mathrm{mM}$ Neocuproine) supplemented with $100 \mu \mathrm{M}$ DFO. The lysates were added to blocking buffer (HEN buffer adjust to $2.5 \%$ SDS and $20 \mathrm{mM} \mathrm{MMTS}$ at $50^{\circ} \mathrm{C}$ for $20 \mathrm{~min}$ with frequent vortexing. The MMTS was then removed by acetone and the proteins were precipitated at $-20^{\circ} \mathrm{C}$ for 20 min. Persulfide in proteins is then pull-downed with thiol reactive beads. S-sulfhydrated proteins are eluted from beads with TCEP treatment following detection by BSA method with anti-GAPDH antibody.
A

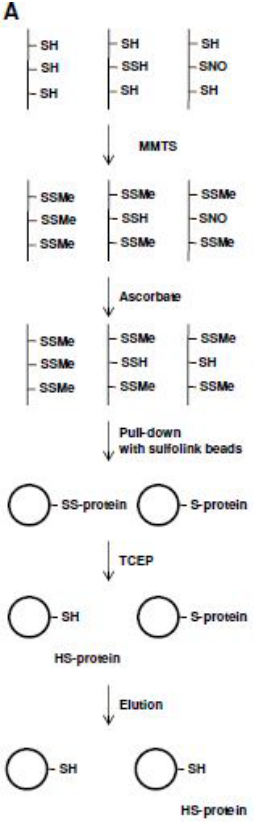

B

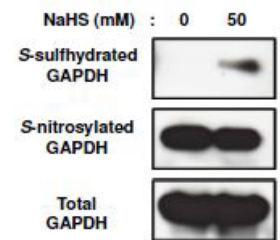

C

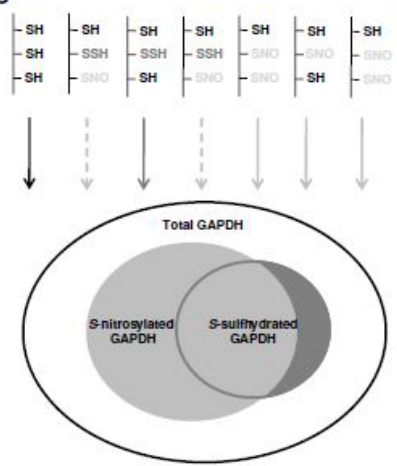

Figure 7: strategies to selectively detect S-sulfhydration. for the detection of $S$-sulfydration. This technique is convenient for the detection of global $S$-sulfhydrated proteins in biological samples. Furthermore, it is able to detect specific $S$-sulfhydrated proteins once protein specific anti-SSH antibody is developed based on the specific amino acid sequence of proteins around $S$-sulfhydration region, like a phospho-antibody. Anti-SSH antibody for specific protein will be a powerful tool for the detection of protein $S$-sulfhydration, in consideration its easiness and convenience compared to other techniques.

\section{New strategies to differentiate protein $S$-sulfhydration and $S$ - nitrosylation}

As shown in Figures 7 and 8, we proposed new strategies to selectively detect $S$-sulfhydration and $S$-nitrosylation in the same sample. The thiol reactive beads can be used for the detection of $S$-sulfhydration and $S$-nitrosylation (Figure 7A). Free thiols are blocked by MMTS and $\mathrm{SNO}$ is reduced by ascorbate. Thiols from SNO and persulfides are pulldowned with thiol reactive beads. The proteins containing disulfide bond from persulfides are eluted with TCEP. Remained proteins bound to beads are eluted which are derived from $S$-nitrosylation. We tested this protocol with GAPDH and visualized with immunoblotting (Figure 7B). S-sulfhydration of GAPDH is significantly increased by NaHS $(50 \mu \mathrm{M})$ in HepG2 cells, while $S$-nitrosylation of GAPDH is slightly decreased. $S$-nitrosylated GAPDH is dominant in samples compared to $S$-sulfhydration. Probably, this is attributed to the different number of cysteine residues which can be $S$-nitrosylated or $S$-sulfhydrated (Figure 7C). GAPDH posseses only one cysteine residue (C152) for $S$-sulfhydration. However, two cysteine residues (C152 and C247 in human GAPDH) were reported for S-nitrosylation of GAPDH [40]. S-sulfhydrated proteins can be captured by thiol which is derived from $S$-nitrosylation even though disulfide bonds are broken by TCEP. Therefore, only pure $S$-sulfhydrated proteins are eluted from beads and detected. Because both $S$-sulfhydrated and $S$-nitrosylated proteins are recognized as $S$-nitrosylated proteins in the above method (Figure 7), we proposed other strategies for the detection of $S$-sulfhydration and $S$-nitrosylation. First, the detection method to measure the released NO and $\mathrm{H}_{2} \mathrm{~S}$ can be employed (Figure $8 \mathrm{~A}$ ). $\mathrm{NO}$ is reduced by maleimide and free thiols are blocked. $\mathrm{NO}$ is then measured by $\mathrm{NO}$ reactive probe which is activated by $\mathrm{NO}$ binding, such as 1,2- diaminoanthraquinone. Thiol-maleimide is released by disulfide reducing reagents which is not contained sulfur. Released thiol-maleimide from $S$-sulfhydration is detected by thiol reactive probe which is activated by $\mathrm{H}_{2} \mathrm{~S}$ binding, like a 7-fluorobenz-2-oxa-1,3-diazole-4-sulfonamide. Second, the combination with thiol- and SNO-reactive probes can be applied for the detection of protein $S$-sulfhydration and $S$-nitrosylation (Figure 8B). Free thiols in proteins are blocked by MMTS and organophosphinebased probe is then incubated to binding SNO. Thiol-reactive probe can be used for the detection of $S$-sulfhydration. The fluorescence-tagged probes, such as organophosphine-based probe and thiol-reactive probe, can be employed to differentiate $S$-sulfhydration and $S$-nitrosylation.

\section{Prospection}

Protein $S$-sulfhydration is just newly discovered and the knowledge on its formation, regulation, and reversion as well as interaction with protein $S$-nitrosylation is still unknown. There are many controversy observations on protein $S$-sulfhdyration, and it is still a matter of debate. One big concern is that $\mathrm{H}_{2} \mathrm{~S}$ may hardly interact with the free thiol group in cysteine to form hydropersulfide. The thiol group would easily react with ROS species to generate sulfenic acid (SOH), and then $\mathrm{H}_{2} \mathrm{~S}$ can readily interact with $\mathrm{SOH}$ to form a persulfide bond. So advanced technique to detect the intermediate products of $\mathrm{H}_{2} \mathrm{~S}$ post- 
Citation: Ju Y, Fu M, Wu L, Yang G (2015) Strategies and Tools for Detection of Protein S-Nitrosylation and S-Sulfhydration. Biochem Anal Biochem 4: 224. doi:10.4172/2161-1009.1000224

A

\begin{tabular}{|c|c|c|c|c|c|c|c|}
\hline -SNO & & -S-Mal & $\sim \mathrm{NO}$ & -S-Mal & & & \\
\hline$-\mathrm{SH}$ & Maleimide & - S-Mal & NO detection & - S-Mal & & $\begin{array}{l}\text { Thiol reactive } \\
\text { probe }\end{array}$ & \\
\hline $\begin{array}{l}-S S H \\
-S \\
1 \\
-S\end{array}$ & & $\begin{array}{l}\text { - SS-Mal } \\
- \text { s } \\
1 \\
-s\end{array}$ & $\longrightarrow$ TCEP & $\begin{array}{l}-\mathrm{SH} \\
-\mathrm{SH} \\
-\mathrm{SH}\end{array}$ & $\sim \mathrm{HS}-\mathrm{Mal}$ & & Probe-S-Mal \\
\hline
\end{tabular}

B

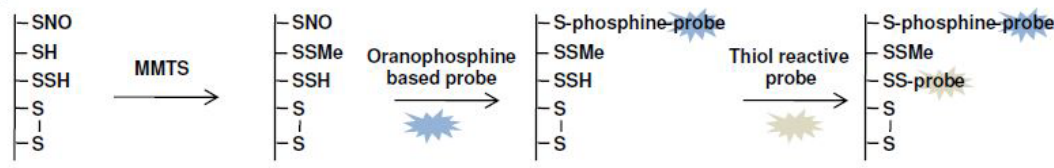

Figure 8: strategies to selectively detect S-nitrosylation.

translationally modification of protein would provide new insight into $\mathrm{H}_{2} \mathrm{~S}$ signaling in both health and diseases. In addition, development of new strategies to detect both $S$-nitrosylation and $S$-sulfhydration in the same samples will provide strong tools to understand the physiological roles of both $\mathrm{NO}$ and $\mathrm{H}_{2} \mathrm{~S}$.

\section{References}

1. Mustafa AK, Gadalla MM, Sen N, Kim S, Mu W, et al. (2009) H2S signals through protein S-sulfhydration. Sci Signal 2: 72

2. Yang G, Zhao K, Ju Y, Mani S, Cao Q, et al. (2013) Hydrogen sulfide protects against cellular senescence via S-sulfhydration of Keap1 and activation of Nrf2. Antioxid Redox Signal 18: 1906-1919.

3. Mustafa AK, Sikka G, Gazi SK, Steppan J, Jung SM,et al. (2011) Hydrogen sulfide as endothelium-derived hyperpolarizing factor sulfhydrates potassium channels. Circ Res. 109: 1259-1268.

4. Zhao K, Ju Y, Li S, Altaany Z, Wang R, et al. (2014) S-sulfhydration of MEK1 leads to PARP-1 activation and DNA damage repair. EMBO Rep 15: 792-800.

5. Altaany Z, Ju Y, Yang G, Wang R (2014) The coordination of S-sulfhydration S-nitrosylation, and phosphorylation of endothelial nitric oxide synthase by hydrogen sulfide. Sci Signal 7: ra87.

6. Liu Y, Yang R, Liu X, Zhou Y, Qu C, et al. (2014) Hydrogen sulfide maintains mesenchymal stem cell function and bone homeostasis via regulation of $\mathrm{Ca}(2+)$ channel sulfhydration. Cell Stem Cell, 15: 66-78.

7. Mazza R, Pasqua T, Cerra MC, Angelone T, Gattuso A (2011) Akt/eNOS signaling and PLN Ssulfhydration are involved in H2S-dependent cardiac effects in frog and rat. Am J Physiol Regul Integr Comp Physiol. 305: 443-451.

8. Sen N, Paul BD, Gadalla MM, Mustafa AK, Sen T, et al. (2012) Hydrogen sulfide-linked sulfhydration of NF-î́B mediates its antiapoptotic actions. Mol Cell 45: 13-24

9. Vandiver MS, Paul BD, Xu R, Karuppagounder S, Rao F, et al. (2013) Sulfhydration mediates neuroprotective actions of parkin. Nat Commun 4 : 1626.

10. Xie ZZ, Shi MM, Xie L, Wu ZY, Li G, et al. (2014) Sulfhydration of p66Shc at cysteine59 mediates the antioxidant effect of hydrogen sulfide. Antioxid Redox Signal 21: 2531-2542.

11. Jaffrey SR, Snyder SH (2001) The biotin switch method for the detection of S-nitrosylated proteins. Sci STKE 2001: pl1.

12. Tang LJ, Li C, Hu SQ, Wu YP, Zong YY, et al. (2012) S-nitrosylation of c-Src via NMDAR-nNOS module promotes c-Src activation and NR2A phosphorylation in cerebral ischemia/reperfusion. Mol Cell Biochem 365: 363-377.

13. Suh KS, Malik M, Shukla A, Ryscavage A, Wright L, et al. (2012) CLIC4 is a tumor suppressor for cutaneous squamous cell cancer. Carcinogenesis. 33 986-995

14. Tanaka K, Shimizu T, Lu L, Yokotani K (2012) Possible involvement of S-nitrosylation of brain cyclooxygenase-1 in bombesin-induced centra activation of adrenomedullary outflow in rats. Eur J Pharmacol. 679: 40-50.

15. Dunne KA, Allam A, McIntosh A, Houston SA, Cerovic V, et al. (2013) Increased
S-nitrosylation and proteasomal degradation of caspase-3 during infection contribute to the persistence of adherent invasive Escherichia coli (AIEC) in immune cells. PLoS One. 8: 68386

16. Liu M, Talmadge JE, Ding SJ (2012) Development and application of sitespecific proteomic approach for study protein S-nitrosylation. Amino Acids. 42 1541-1551.

17. Murray $\mathrm{Cl}$, Uhrigshardt H, O'Meally RN, Cole RN, Van Eyk JE (2012) Identification and quantification of S-nitrosylation by cysteine reactive tandem mass tag switch assay. Mol Cell Proteomics 11: 111.

18. Qu Z, Meng F, Bomgarden RD, Viner RI, Li J, et al (2014) Proteomic quantification and site-mapping of S-nitrosylated proteins using isobaric iodoTMT reagents. J Proteome Res. 13: 3200-3211.

19. Faccenda A, Bonham CA, Vacratsis PO, Zhang X, Mutus B (2010) Gold nanoparticle enrichment method for identifying S-nitrosylation and S-glutathionylation sites in proteins. J Am Chem Soc 132: 11392-11394.

20. Zhang HH, Feng L, Wang W, Magness RR, Chen DB (2012) Estrogenresponsive nitroso-proteome in uterine artery endothelial cells: role of endothelial nitric oxide synthase and estrogen receptor- $\hat{I}^{2}$. J Cell Physiol 227 146-159.

21. Maiti D, Sarkar TS, Ghosh S. Detection of S-nitrosothiol and nitrosylated proteins in Arachis hypogaea functional nodule: response of the nitrogen fixing symbiont. PLoS One. 7: 45526.

22. Torta F, Elviri L, Bachi A (2010) Direct and indirect detection methods for the analysis of S-nitrosylated peptides and proteins. Methods Enzymol 473: 265280.

23. Doulias PT, Tenopoulou M, Greene JL, Raju K, Ischiropoulos HI (2013) Nitric oxide regulates mitochondrial fatty acid metabolism through reversible protein S-nitrosylation. Sci Signal. 6: 1

24. Wang H, Fu L, Zhang $X$ (2011) Comparison of expression, purification and characterization of a new pectate lyase from Phytophthora capsici using two different methods. BMC Biotechnol 11: 32

25. Boullerne Al, Rodriguez JJ, Touil T, Brochet B, Schmidt S, et al. (2002) Anti-S-nitrosocysteine antibodies are a predictive marker for demyelination in experimental autoimmune encephalomyelitis: implications for multiple sclerosis. J Neurosci. 22: 123-132.

26. Greco TM, Hodara R, Parastatidis I, Heijnen HF, Dennehy MK, et al (2006) Identification of S-nitrosylation motifs by site-specific mapping of the S-nitrosocysteine proteome in human vascular smooth muscle cells. Proc Nat Acad Sci U S A. 103: 7420-7425.

27. Sangwung P, Greco TM, Wang Y, Ischiropoulos H, Sessa WC, et al. (2012) Proteomic identification of S-nitrosylated Golgi proteins: new insights into endothelial cell regulation by eNOS-derived NO. PLoS One 7: e31564.

28. Wang X, Liu W, Wu Y, Liu X, Liang X, et al. (2013) C-reactive protein reduces protein S-nitrosylation in endothelial cells. Mol Cell Biochem 375: 131-138.

29. Wang Y, Liu T, Wu C, Li H (2008) A strategy for direct identification of protein S-nitrosylation sites by quadrupole time-of-flight mass spectrometry. J Am Soc Mass Spectrom 19: 1353-1360.

30. Lamotte O, Bertoldo JB, Besson-Bard A, Rosnoblet C, Aimé S, et al. (2015) 
Citation: Ju Y, Fu M, Wu L, Yang G (2015) Strategies and Tools for Detection of Protein S-Nitrosylation and S-Sulfhydration. Biochem Anal Biochem 4: 224. doi:10.4172/2161-1009.1000224

Protein S-nitrosylation: specificity and identification strategies in plants. Front Chem 2: 114

31. Kaneko R, Wada Y (2013) Decomposition of protein nitrosothiolsin matrixassisted laserdesorption/ionization and electrospray ionization mass spectrometry. J Mass Spectrom. 38: 526-530.

32. Kuncewicz T, Sheta EA, Goldknopf IL, Kone BC (2003) Proteomic analysis of S-nitrosylated proteins in mesangial cells. Mol Cell Proteomics 2: 156-163.

33. Mustafa AK, Gadalla MM, Sen N, Kim S, Mu W, et al. (2009) H2S signals through protein S-sulfhydration. Sci Signal 2: ra72.

34. Pan J, Carroll KS (2013) Persulfide reactivity in the detection of protein s-sulfhydration. ACS Chem Biol 8: 1110-1116.

35. Ju Y, Untereiner A, Wu L, Yang G (2015) H2S-induced S-sulfhydration of pyruvate carboxylase contributes to gluconeogenesis in liver cells. Biochim Biophys Acta. 1850: 2293-2303.
36. Zhang D, Macinkovic I, Devarie-Baez NO, Pan J, Park CM, et al. (2014) Detection of protein S-sulfhydration by a tag-switch technique. Angew Chem Int Ed Engl 53: 575-581.

37. Kashiba-Iwatsuki M, Kitoh K, Kasahara E, Yu H, Nisikawa M, et al (1997) Ascorbic acid and reducing agents regulate the fates and functions of S-nitrosothiols. J Biochem. 122: 1208-1214.

38. Peng B, Xian M (2015) Hydrogen sulfide detection using nucleophilic substitution-cyclization-based fluorescent probes. Methods Enzymol 554: 4762.

39. Yu Q, Zhang KY, Liang H, Zhao Q, Yang T, et al. (2015) Dual-emissive nanohybrid for ratiometric luminescence and lifetime imaging of intracellular hydrogen sulfide. ACS Appl Mater Interfaces. 7: 5462-5470.

40. Jia J, Arif A, Willard B, Smith JD, Stuehr DJ, et al. (2012) Protection of extraribosomal RPL13a by GAPDH and dysregulation by S-nitrosylation. Mol Cell 47: 656-663 